\title{
Lipoprotein Lipase and PPAR Alpha Gene Polymorphisms, Increased Very-Low-Density Lipoprotein Levels, and Decreased High-Density Lipoprotein Levels as Risk Markers for the Development of Visceral Leishmaniasis by Leishmania infantum
}

\author{
Márcia Dias Teixeira Carvalho, ${ }^{1}$ Diego Peres Alonso, ${ }^{2}$ Célia Maria Vieira Vendrame, ${ }^{1}$ \\ Dorcas Lamounier Costa, ${ }^{3}$ Carlos Henrique Nery Costa, ${ }^{3}$ Guilherme Loureiro Werneck, ${ }^{4,5}$ \\ Paulo Eduardo Martins Ribolla, ${ }^{2}$ and Hiro Goto ${ }^{1,6}$ \\ ${ }^{1}$ Laboratório de Soroepidemiologia e Imunobiologia, Instituto de Medicina Tropical de São Paulo, Universidade de São Paulo, \\ Avenida Dr. Enéas de Carvalho Aguiar 470, Prédio II, $4^{\circ}$ Andar, 05403-000 São Paulo, SP, Brazil \\ ${ }^{2}$ Departamento de Parasitologia, Instituto de Biociências, Universidade Estadual Paulista, 18618-970 Botucatu, SP, Brazil \\ ${ }^{3}$ Instituto de Doenças Tropicais Natan Portella, Universidade Federal do Piauí, 64001-450 Teresina, PI, Brazil \\ ${ }^{4}$ Departamento de Epidemiologia, Instituto de Medicina Social, Universidade do Estado do Rio de Janeiro, \\ 20550-900 Rio de Janeiro, RJ, Brazil \\ ${ }^{5}$ Instituto de Estudos em Saúde Coletiva, Universidade Federal do Rio de Janeiro, 21941-598 Rio de Janeiro, RJ, Brazil \\ ${ }^{6}$ Departamento de Medicina Preventiva, Faculdade de Medicina, Universidade de São Paulo, 01246-903 São Paulo, SP, Brazil
}

Correspondence should be addressed to Hiro Goto; hgoto@usp.br

Received 22 May 2014; Accepted 2 August 2014; Published 27 August 2014

Academic Editor: Christophe Chevillard

Copyright (C) 2014 Márcia Dias Teixeira Carvalho et al. This is an open access article distributed under the Creative Commons Attribution License, which permits unrestricted use, distribution, and reproduction in any medium, provided the original work is properly cited.

\begin{abstract}
In visceral leishmaniasis (VL) endemic areas, a minority of infected individuals progress to disease since most of them develop protective immunity. Therefore, we investigated the risk markers of VL within nonimmune sector. Analyzing infected symptomatic and, asymptomatic, and noninfected individuals, VL patients presented with reduced high-density lipoprotein cholesterol (HDLC), elevated triacylglycerol (TAG), and elevated very-low-density lipoprotein cholesterol (VLDL-C) levels. A polymorphism analysis of the lipoprotein lipase (LPL) gene using HindIII restriction digestion $(N=156$ samples) $(\mathrm{H}+=$ the presence and $\mathrm{H}-$ $=$ the absence of mutation) revealed an increased adjusted odds ratio $(\mathrm{OR})$ of $\mathrm{VL}$ versus noninfected individuals when the $\mathrm{H}+/ \mathrm{H}+$ was compared with the $\mathrm{H}-/ \mathrm{H}-$ genotype $(\mathrm{OR}=21.3 ; 95 \% \mathrm{CI}=2.32-3335.3 ; P=0.003)$. The $\mathrm{H}+/ \mathrm{H}+$ genotype and the $\mathrm{H}+$ allele were associated with elevated VLDL-C and TAG levels $(P<0.05)$ and reduced HDL-C levels $(P<0.05)$. An analysis of the L162V polymorphism in the peroxisome proliferator-activated receptor alpha (PPAR $\alpha)$ gene $(n=248)$ revealed an increased adjusted OR when the Leu/Val was compared with the Leu/Leu genotype $(\mathrm{OR}=8.77 ; 95 \% \mathrm{CI}=1.41-78.70 ; P=0.014)$. High TAG $(P=0.021)$ and VLDL-C $(P=0.023)$ levels were associated with susceptibility to VL, whereas low HDL $(P=0.006)$ levels with resistance to infection. The mutated LPL and the PPAR $\alpha$ Leu/Val genotypes may be considered risk markers for the development of VL.
\end{abstract}

\section{Introduction}

Visceral leishmaniasis (VL) is a systemic disease that is prevalent in tropical and subtropical regions. VL is caused by various species of protozoa within the genus Leishmania and of the Leishmania donovani complex, including Leishmania infantum (previously referred to as L. chagasi), in the new world [1]. In a VL-endemic area, the most infected individuals remain asymptomatic. Individuals with infection may present 
with mild symptoms and a minority of infected individuals, approximately $10-20 \%$, progress to active severe disease that is characterized by fever, hepatosplenomegaly, pancytopenia, hypergammaglobulinemia, and severe weight loss [2, 3]. However, the factors that are associated with susceptibility to infection progression are not well known.

The immune system has been the natural focus of previous studies that assessed the risk markers for VL development. Most studies have focused on genes that are related to the immune system. In studies with approaches that were based mainly on family groups with VL infection, the SLC11A1 (formerly called as the natural resistance-associated macrophage protein-1, NRamp-1) gene on chromosome 2q35 $[4,5]$, interleukin-4/interleukin-9 [6], the TNF locus [7], a locus on chromosome 22q12 [8, 9], IL-18 [10], and IL-1beta [11] polymorphisms have been associated with VL infection. However, in their 2009 review [12], Blackwell et al. suggested that most of these polymorphisms had marginal effects on infection progression. Recently, the IL-10 $819 \mathrm{C} / \mathrm{T}$ genotype was associated with susceptibility to VL development in Iran [13]. In well-powered population-based studies, the CXCR2 gene [14] and DLL1, which encode delta-like 1, the ligand for Notch 3 [15], were associated with susceptibility to VL development but not SLC11A1 in India [16]. More recently, another well-powered population-based study revealed a consistent contribution of the HLA-DRB1-HLA-DQA1 HLA class II region to $\mathrm{VL}$ susceptibility in the Indian subcontinent and Brazil [17]. However, the role of these genetic determinants in the pathogenesis of VL was not addressed in these studies.

In L. infantum infection, protective immunity develops in the majority of individuals who reside in VL-endemic areas [2]. During active disease, immune derangement occurs; however, the immune system remains highly activated [3]. Therefore, nonspecific factors may play important roles in infections by parasites that can efficiently evade effective immune responses. Furthermore, a study has indicated that factors not related to acquired cell-mediated immunity may explain the progression of VL [18]. Therefore, in this study, we focused on nonimmune sector, that is, lipid metabolism.

Several studies have reported on lipid alterations in human VL cases [19-22]. In this study, we initially analyzed serum-lipid profiles in a large number of patients with clinical manifestations of VL. We found high triacylglycerol (TAG) levels, high very-low-density lipoprotein cholesterol (VLDLC) levels, and low high-density lipoprotein cholesterol (HDLC) levels in infected symptomatic individuals. These alterations may have resulted from the inflammatory infectious process; however, we investigated the possible role of these lipoprotein fractions in susceptibility to L. infantum infection because previous results have suggested that amastigotes are largely dependent on protein and lipids as an energy source within host cells [23]. In addition to the analysis of lipoprotein fraction alterations, we extended the study to analyze the factors that modulate TAG and HDL levels. These additional factors included lipoprotein lipase (LPL), which hydrolyzes TAG from TAG-rich lipoprotein particles in plasma [24] and reduces the uptake of a TAG-rich artificial emulsion [25], apolipoprotein E (apoE), which mediates the binding of lipoprotein particles to receptors in the liver that mediate their clearance [26], and peroxisome proliferatoractivated receptor alpha $(\operatorname{PPAR} \alpha)$, which is involved in lipid metabolism, including roles in oxidation pathways, the uptake and transport of fatty acids, and lipoprotein synthesis routes [27]. Therefore, we focused on LPL, apoE, and PPAR $\alpha$ gene polymorphisms as candidates for genetic risk markers for disease development in L. infantum infection. LPL gene polymorphisms were searched using HindIII and PVuII restriction enzymes known to reveal mutated genes that correlate with TAG level alteration and the former also with $\mathrm{HDL}$ level alteration $[28,29]$. We found that the $\mathrm{H}+/ \mathrm{H}+$ genotype and the $\mathrm{H}+$ allele, which were identified using HindIII restriction in the LPL gene, were more frequent in infected symptomatic individuals and were notably associated with high levels of VLDL-C, TAG, and HDL-C. The apoE gene is presented in three main isoforms (apoE2, apoE3, and apoE4) that are coded by three common alleles (E2, E3, and E4) [26]. We found that the E3/E3 genotype and the E3 allele were more frequent in infected symptomatic individuals than in infected asymptomatic or noninfected individuals, and these factors were associated with high levels of VLDL cholesterol and TAG. We found that the Leu/Val genotype and the Val allele of the L162V polymorphism in the PPAR $\alpha$ gene were more frequent in infected symptomatic individuals than in infected asymptomatic or noninfected individuals but with no apparent association with alterations in lipoprotein levels.

We hypothesized that certain lipid fractions may play a role in the development of $L$. infantum infection. In this study, we found that high levels of triglycerides and VLDL and low levels of HDL favor the development of active disease. Therefore, we suggest that related lipoprotein lipase and apolipoprotein $\mathrm{E}$ genotypes that lead to alterations in these lipoprotein levels as well as the presence of the PPAR $\alpha$ L162V allele may be susceptibility factors and markers of VL development.

\section{Materials and Methods}

2.1. Study Design and Subjects. The individuals who were included in this study were from Teresina, Piaui State, Northeastern Brazil. The inclusion criteria were as follows: unrelated individuals who presented with active VL (referred to as infected symptomatic), healthy individuals who had been infected with $L$. infantum as determined by a positive anti-Leishmania (Montenegro) delayed-type hypersensitivity skin test and/or the presence of anti-Leishmania antibodies (referred to as infected asymptomatic), and healthy individuals who had no evidence of present or past infection with $L$. infantum as determined by both a negative Montenegro skin test and the absence of specific antibodies. The subjects and materials in this study were similar to those in the previous study by Alonso et al. [30] in which possible ethnic and environmental biases were analyzed and deemed not to be confounding factors.

2.2. Ethics Statement. This study was approved by the ethics committees of the participating institutions (Comissão de Ética em Pesquisa da Universidade Federal do Piauí and Comissão de Ética para Análise de Projetos de Pesquisa da 
Diretoria Clínica do Hospital das Clínicas e da Faculdade de Medicina da Universidade de São Paulo). Written informed consent was obtained from all of the participants or their parents or guardians. Additionally, the samples and data were treated anonymously.

2.3. Analysis of Biochemical Parameters. After 12 hours of fasting, venous blood samples were drawn from 81 individuals to measure lipid parameters. The total cholesterol (TC), low-density lipoprotein cholesterol (LDL-C), HDL-C, and TAG concentrations were measured using automated methods (Roche Diagnostics Co., Indianapolis, IN, USA), and the VLDL-C concentrations were calculated (TAG concentration/5) using the Friedewald equation.

2.4. Genetic Analysis of the LPL Polymorphism. DNA was extracted from peripheral blood white cells using the GFX Genomic Blood DNA Purification Kit (GE Healthcare, Piscataway, NJ, USA) and subjected to amplification by polymerase chain reaction (PCR) using a Bio-Rad Laboratories DNA Thermal Cycler (Hercules, CA, USA).

The variations were confirmed using a restriction fragment length polymorphism (RFLP) assay in which the PCRamplified products were digested by HindIII for 156 individuals or by PvuII for 130 individuals. Due to the availability, not all samples were analyzed using both enzymes. One primer set was used to amplify the sequence around a HindIII restriction site in intron 8 (the forward primer was $5^{\prime}$-TTTAGGCCTGAAGTTTCCAC-3', and the reverse primer was $5^{\prime}$-CTCCCTAGAACAGAAGATC- $3^{\prime}$ ) [31]. The amplified fragment was $1.3 \mathrm{~kb}$ in size. The other primer set was obtained from the DNA sequences that flanked a PvuII restriction site in intron 6 (the forward primer was $5^{\prime}$-TAGAGGTTGAGGCACCTGTGC-3', and the reverse primer was $5^{\prime}$-GTGGGTGAATCACCTGAGGTC-3') [32]. The amplified fragment was $858 \mathrm{bp}$ long. The amplification of the region that flanked the HindIII site was performed over 33 cycles at $95^{\circ} \mathrm{C}$ for $1 \mathrm{~min}, 60^{\circ} \mathrm{C}$ for $2 \mathrm{~min}$, and $72^{\circ} \mathrm{C}$ for $2 \mathrm{~min}$. The amplification of the sequence that surrounded the PvuII site was performed over 32 cycles at $95^{\circ} \mathrm{C}$ for $1 \mathrm{~min}, 68^{\circ} \mathrm{C}$ for $2 \mathrm{~min}$, and $72^{\circ} \mathrm{C}$ for $2 \mathrm{~min}$. The PCR-amplified products were digested with HindIII or PvuII. HindIII yielded fragments that were $600 \mathrm{bp}$ and $700 \mathrm{bp}$ long, and PvuII yielded fragments that were $266 \mathrm{bp}$ and $592 \mathrm{bp}$ long [28].

2.5. Genetic Analysis of the apoE Polymorphism. Using extracted DNA, the variations were confirmed by a single-nucleotide polymorphism (SNP) assay in which the PCR-amplified products were analyzed using the SNaPshot Multiplex Kit (Applied Biosystems, Foster City, CA, USA) for 115 individuals. We used one set of primers that were derived from exon 4 in the apoE gene, and PCR was used to amplify a $244 \mathrm{bp}$ region that spanned the E2, E3, and E4 allelic sites (the forward primer was $5^{\prime}$ TAAGCTTGGCACGGCTGTCCAAGGA- $3^{\prime}$, and the reverse primer was $5^{\prime}$-ACAGAATTCGCCCCGGCCTGGTACAC$3^{\prime}$ ) [26]. The amplification of the apoE site was performed with an initial denaturation at $95^{\circ} \mathrm{C}$ for $2 \mathrm{~min}$, which was followed by 35 cycles at $95^{\circ} \mathrm{C}$ for $1 \mathrm{~min}, 60^{\circ} \mathrm{C}$ for
$1 \mathrm{~min}$, and $72^{\circ} \mathrm{C}$ for $30 \mathrm{~s}$. The SNaPshot Multiplex Kit was used to investigate the presence of the E2, E3, and E4 alleles in the amplified products. Two specific primers for apoE SNPs were utilized (the forward primer was $5^{\prime}$ GCGCGGACATGGAGGACGTG- $3^{\prime}$, and the reverse primer was $5^{\prime}$-CCCCCGGCCTGGTACACTGCCAGGC- $3^{\prime}$ ). The $\mathrm{SNaPshot} \mathrm{Multiplex} \mathrm{Kit} \mathrm{reactions} \mathrm{were} \mathrm{performed} \mathrm{in} \mathrm{the} \mathrm{ABI}$ 377 Automatic Sequencer (Applied Biosystems) following the manufacturer's recommendations.

2.6. Genotyping of the PPAR $\alpha$ L162V Polymorphism. Using extracted DNA, the PPAR $\alpha$ gene L162V polymorphism was genotyped by allelic discrimination using TaqMan probes on a custom assay-on-demand basis by Applied Biosystems. A pair of oligos was custom-designed to amplify a fragment that contained the L162V SNP together with different allele-specific fluorescent probes using PCR. Genotyping was performed using a real-time PCR reaction (StepOnePlus Applied Biosystems) according to the following protocol: in a $10 \mu \mathrm{L}$ reaction, $5 \mu \mathrm{L}$ of Maxima Probe/ROX qPCR Master Mix (2X) (Fermentas) was mixed with $0.5 \mu \mathrm{L}$ of TaqMan reagents (a 20X master mix that contained the probes and the pair of oligos) and $4.5 \mu \mathrm{L}$ of autoclaved Milli-Q water. The reaction was performed following the cycling protocol, initial denaturation for $10 \mathrm{~min}$ at $95^{\circ} \mathrm{C}$, followed by 40 cycles of denaturation at $95^{\circ} \mathrm{C}$ for $15 \mathrm{~s}$, and annealing and extension for $60 \mathrm{~s}$ at $60^{\circ} \mathrm{C}$. After cycling, a graph of allelic discrimination was generated.

2.7. Statistical Analysis. The data were expressed as means and standard deviations and compared using ANOVA tests. The Student Newman-Keuls posttest was used for post hoc statistical comparisons among the groups, and the $t$-test was used for statistical comparisons between the groups. The frequencies and comparison distributions of the genotypes and alleles for each polymorphic site were estimated by gene counting. Consistency with the Hardy-Weinberg proportions was tested by the log-likelihood ratio. We used the Pearson $\chi^{2}$ test for trends to analyze differences in the frequency of the LPL genotypes and alleles between the outcome groups (infected symptomatic, infected asymptomatic, and noninfected). Associations between levels of lipids and different genotypes and the patient outcomes were expressed as odds ratios (OR) and their respective 95\% confidence intervals (95\% CI), adjusted for age and gender, estimated by means of exact logistic regression models. Models compared the infected (both symptomatic and asymptomatic) individuals to the noninfected individuals. The levels of lipids were analyzed both as continuous and dichotomous variables. For the dichotomous analyses, the cut-off points used for TC, LDL-C, HDL-C, TAG, and VLDL-C were established by selecting the point of the ROC curve that maximized the sensitivity and specificity for separating noninfected and infected symptomatic individuals. Data were analyzed using Stata statistical software (Stata for Windows 13.0, StataCorp, College Station, TX), Systat version 10 (Point Richmond, Richmond, CA), and GraphPad Prism 3.0 (GraphPad Software Inc., San Diego, CA, USA). A $P$ value $<0.05$ was considered to be significant. 
TABLE 1: Levels of serum lipids in individuals exposed to Leishmania infantum from endemic area.

\begin{tabular}{lcccccc}
\hline \multirow{2}{*}{ Lipids } & \multicolumn{2}{c}{ Infected symptomatic individuals } & \multicolumn{2}{c}{ Infected asymptomatic individuals } & \multicolumn{2}{c}{ Noninfected individuals } \\
& $n$ & $\mathrm{mg} / \mathrm{dL}$ & $n$ & $\mathrm{mg} / \mathrm{dL}$ & $n$ & $\mathrm{mg} / \mathrm{dL}$ \\
\hline TC & 17 & $121.2 \pm 34.6$ & 28 & $143.0 \pm 42.1$ & 36 & $139.9 \pm 36.1$ \\
LDL-C & 17 & $59.5 \pm 30.8$ & 28 & $85.5 \pm 42.2$ & 36 & $77.1 \pm 36.0$ \\
HDL-C & 17 & $38.3 \pm 4.3^{*}$ & 28 & $39.0 \pm 5.8^{*}$ & 36 & $43.0 \pm 7.2$ \\
TAG & 17 & $219.4 \pm 84.9^{* \dagger}$ & 28 & $169.1 \pm 62.7$ & 36 & $157.9 \pm 67.6$ \\
VLDL-C & 17 & $43.8 \pm 16.9^{*, \dagger}$ & 28 & $33.9 \pm 12.4$ & 36 & $31.6 \pm 13.5$ \\
\hline
\end{tabular}

$\mathrm{TC}=$ total cholesterol; LDL-C = low-density lipoprotein cholesterol; HDL-C = density lipoprotein cholesterol; TAG = triacylglycerol; VLDL $=$ very-low-density lipoprotein cholesterol. Lipid values expressed as the mean \pm SD. $P$ value analyzed by ANOVA test with the Student Newman-Keuls posttest. ${ }^{*} P<0.05$ versus noninfected individuals; ${ }^{\dagger} P<0.05$ versus infected asymptomatic individuals. $P$ values in bold indicate statistically significant differences.

\section{Results}

In this study, using a candidate gene approach to reveal the genetic traits and the resulting phenotypic expressions, that is, lipoprotein levels, we aimed to identify the risk markers for the development of $L$. infantum infection.

The assessment of the lipid fraction levels revealed elevated triacylglycerol (TAG) levels and elevated very-lowdensity lipoprotein cholesterol (VLDL-C) levels in all of the infected symptomatic individuals compared with the infected asymptomatic and noninfected individuals $(P<$ 0.05) (Table 1) (according to the III Brazilian Guidelines on Dyslipidemia [33], the optimal TAG level is $<130 \mathrm{mg} / \mathrm{dL}$ for individuals up to 20 years of age and $<150 \mathrm{mg} / \mathrm{dL}$ in individuals who are older than 20 years of age). Because the ideal age-matched endemic controls for this approach could not be obtained due to technical and ethical reasons, the average age (mean \pm standard deviation) of the infected symptomatic individuals $(n=17 ; 6.2 \pm 7.6$ years of age) was significantly lower than the average ages of the infected asymptomatic ( $n=28 ; 30.2 \pm 12.4$ years of age) and noninfected individuals $(n=36 ; 27.9 \pm 19.2$ years of age). To circumvent bias, we separately examined the individuals who were younger than 20 years of age when the differences between the groups were still maintained (data not shown). Additionally, we observed reduced high-density lipoprotein cholesterol (HDL-C) levels in both the infected symptomatic and asymptomatic individuals compared with the noninfected individuals $(P<0.05)$ (Table 1$)$.

We analyzed the polymorphisms of the LPL, apoE, and $\operatorname{PPAR} \alpha$ genes. The genotype distributions for all of the polymorphisms were in Hardy-Weinberg equilibrium for all of the groups (Table 2). Significant differences were observed in the frequencies of the polymorphic HindIII-restricted genotypes $(\mathrm{H}+/ \mathrm{H}+$ versus $\mathrm{H}+/ \mathrm{H}-, P<0.05 ; \mathrm{H}+/ \mathrm{H}+$ versus $\mathrm{H}-/ \mathrm{H}-, P<0.01)$ and the HindIII-restricted alleles $(\mathrm{H}+$ versus $\mathrm{H}-, P<0.01$ ) (Table 1$)$. However, no differences were detected between the groups according to the frequencies of either the genes or the alleles of the PvuII restriction products (Table 2). In the apoE gene, the analysis of the three common apoE alleles (E2, E3, and E4) that code the three main apoE isoforms (apoE2, apoE3, and apoE4) indicated that the E3/E3 genotype was the most frequent, followed by the E3/E4, E2/E3, E2/E4, and E4/E4 genotypes. The $\mathrm{E} 2 / \mathrm{E} 2$ genotype was not detected in any of the individuals.
In the analysis of the apoE alleles, the E3 allele was the most frequent, followed by the E4 and E2 alleles. Significant differences were observed between the frequency of the E4 allele and that of the E3 allele $(P<0.05)$ (Table 2). We found that the E3/E3 genotype and the E3 allele were more frequent in infected symptomatic individuals than in infected asymptomatic or noninfected individuals (Table 2). In the PPAR $\alpha$ gene L162V polymorphism, we found significant differences in the frequencies of the Leu/Leu and Leu/Val $(P<0.05)$ genotypes and the Leu and Val alleles $(P<0.01)$ between the groups (Table 2).

In the analysis of the association between the genotypes and the lipoprotein levels in all of the individuals regardless of the outcome, the TAG and VLDL-C levels were significantly higher in the $\mathrm{H}+/ \mathrm{H}+$ individuals $(P<0.01)$ (Table 3 ). The individuals with the $\mathrm{H}+/ \mathrm{H}+$ genotype had significantly lower HDL-C levels than the individuals with the $\mathrm{H}+/ \mathrm{H}-$ genotype $(P<0.05)$. The serum-lipid levels were measured in only two $\mathrm{H}-/ \mathrm{H}-$ individuals, which was insufficient for the statistical analysis (the means are presented in the legend of Table 3 ). In the individuals with the P1/P1 genotype, no significant differences in the lipid profiles were observed between the P1/P2 and P2/P2 individuals (Table 3 ). In the analysis of the apoE genotypes and alleles, we found that the TAG and VLDL-C levels in the individuals with the E2/E3 genotype were significantly higher than those in the E3/E3 and E3/E4 $(P<0.05)$ individuals (Table 3$)$. The serum-lipid data were available in only one E4/E4 individual (all of the variables are shown in the legend of Table 3 ). The serum-lipid levels were not evaluated in the E2/E4 and E2/E2 individuals. Furthermore, the TAG and VLDL-C levels were significantly higher in the E2 individuals than in the E3 and E4 individuals $(P<0.05)$, but the TC levels were higher in the E3 individuals than in the E2 and E4 individuals $(P<0.05)$ (Table 3$)$. The E3 allele was associated with higher TC levels than with the E2 and E4 alleles; however, the levels were still optimal [33]. The lipid profiles were not associated with either the genotypes or the alleles of the L162V polymorphism in the PPAR $\alpha$ gene (Table 3).

To analyze the association between the genotypic and phenotypic expressions and the outcomes, we performed a multivariate analysis to calculate the adjusted odds ratio (OR) because of the significant differences in the ages and gender distribution among the studied groups. The results of this 
TABLE 2: Frequency of genotypes and alleles of LPL, apoE, and PPAR $\alpha$ genes in individuals exposed to Leishmania infantum.

\begin{tabular}{|c|c|c|c|c|c|c|c|c|}
\hline & \multirow{2}{*}{ Polymorphisms $^{\S \S}$} & \multicolumn{2}{|c|}{ Infected symptomatic individuals } & \multicolumn{2}{|c|}{ Infected asymptomatic individuals } & \multicolumn{2}{|c|}{ Noninfected individuals } & \multirow{2}{*}{$P$ value } \\
\hline & & $n$ & $\%$ of total & $n$ & $\%$ of total & $n$ & $\%$ of total & \\
\hline \multirow{6}{*}{ HindIII } & $\mathrm{H}+/ \mathrm{H}+{ }^{(\mathrm{a})}$ & 24 & 46.2 & 17 & 35.4 & 13 & 23.2 & $0.040^{\text {(a vs. b) }}$ \\
\hline & $\mathrm{H}+/ \mathrm{H}-{ }^{(b)}$ & 26 & 50.0 & 27 & 56.3 & 34 & 60.7 & $0.103^{\text {(b vs. c) }}$ \\
\hline & $\mathrm{H}-/ \mathrm{H}-{ }^{(\mathrm{c})}$ & 2 & 3.8 & 4 & 8.3 & 9 & 16.1 & $0.006^{(\text {a vs. c) }}$ \\
\hline & & $\mathrm{HWE}^{\dagger}$ & 0.116 & & 0.138 & & 0.099 & \\
\hline & $\mathrm{H}+{ }^{(\mathrm{d})}$ & 74 & 71.5 & 61 & 63.5 & 60 & 53.6 & \\
\hline & $\mathrm{H}-{ }^{(\mathrm{e})}$ & 30 & 28.8 & 35 & 36.5 & 52 & 46.4 & $\mathbf{0 . 0 0 8}^{\text {(d vs. e) }}$ \\
\hline \multirow{6}{*}{ PvuII } & $\mathrm{P} 1 / \mathrm{Pl}^{(\mathrm{f})}$ & 12 & 28.6 & 13 & 29.5 & 16 & 37.2 & $0.429^{(\mathrm{f} \text { vs. g) }}$ \\
\hline & $\mathrm{P} 1 / \mathrm{P} 2^{(\mathrm{g})}$ & 25 & 59.5 & 27 & 61.4 & 23 & 53.5 & $0.835^{\text {(g vs. } h)}$ \\
\hline & $\mathrm{P} 2 / \mathrm{P} 2^{(\mathrm{h})}$ & 5 & 11.9 & 4 & 9.1 & 4 & 9.3 & $0.507^{(\mathrm{f} \text { vs. h })}$ \\
\hline & & $\mathrm{HWE}^{\dagger}$ & 0.146 & & 0.062 & & 0.294 & \\
\hline & $P 1^{(i)}$ & 49 & 58.3 & 53 & 60.2 & 55 & 64.0 & \\
\hline & $\mathrm{P} 2^{(\mathrm{j})}$ & 35 & 41.7 & 35 & 39.8 & 31 & 36.0 & $0.452^{(\mathrm{i} \text { vs. } \mathrm{j})}$ \\
\hline \multirow{8}{*}{ ApoE } & $\mathrm{E} 2 / \mathrm{E} 3^{(\mathrm{k})}$ & 3 & 8.1 & 4 & 10.5 & 2 & 5.0 & $0.833^{\text {(k vs. l) }}$ \\
\hline & $\mathrm{E} 3 / \mathrm{E} 3^{(1)}$ & 30 & 81.1 & 24 & 63.2 & 26 & 65.0 & $0.116^{(1 \text { vs. } m)}$ \\
\hline & $\mathrm{E} 3 / \mathrm{E} 4^{(\mathrm{m})}$ & 4 & 10.8 & 10 & 26.3 & 10 & 25.0 & $0.214^{\text {(k vs. m) }}$ \\
\hline & E2/E4 & 0 & 0.0 & 0 & 0.0 & 1 & 2.5 & \\
\hline & $\mathrm{E} 4 / \mathrm{E} 4$ & 0 & 0.0 & 0 & 0.0 & 1 & 2.5 & \\
\hline & $\mathrm{E} 2^{(\mathrm{n})}$ & 3 & 4.1 & 4 & 5.3 & 3 & 3.7 & $0.953^{\text {(n vs. o) }}$ \\
\hline & $\mathrm{E} 3^{(\mathrm{o})}$ & 67 & 90.5 & 62 & 81.6 & 64 & 80.0 & $\mathbf{0 . 0 3 8 ^ { ( o ~ v s . ~ p ) ~ }}$ \\
\hline & $\mathrm{E} 4^{(\mathrm{p})}$ & 4 & 5.4 & 10 & 13.1 & 13 & 16.3 & $0.230^{\text {(n vs. } p)}$ \\
\hline \multirow{6}{*}{$\operatorname{PPPAR} \alpha$} & Leu/Leu ${ }^{(\mathrm{q})}$ & 48 & 80.0 & 61 & 87.1 & 92 & 93.9 & $0.022^{\text {(q vs. r) }}$ \\
\hline & Leu/Val ${ }^{(\mathrm{r})}$ & 11 & 18.3 & 8 & 11.4 & 6 & 6.1 & \\
\hline & Val/Val & 1 & 1.7 & 1 & 1.4 & 0 & 0.0 & \\
\hline & & $\mathrm{HWE}^{\dagger}$ & 0.693 & & 0.247 & & 0.745 & \\
\hline & $\mathrm{Leu}^{(s)}$ & 107 & 89.2 & 130 & 92.9 & 190 & 96.9 & \\
\hline & $\mathrm{Val}^{(\mathrm{t})}$ & 13 & 10.8 & 10 & 7.1 & 6 & 3.1 & $\mathbf{0 . 0 0 8}^{\text {(s vs. t) }}$ \\
\hline
\end{tabular}

${ }^{\$ S} P$ value analyzed by $\chi^{2}$ test for trend; ${ }^{\dagger} P$ value in Hardy-Weinberg equilibrium (HWE).

Note: $\mathrm{H}+$ indicates presence and $\mathrm{H}-$ indicates absence of the HindIII restriction site. P1 indicates absence and P2 indicates presence of the PvuII restriction site. Apo E polymorphism results in six genotypes (E2/E2, E2/E3, E2/E4, E3/E3, E3/E4, and E4/E4). E2/E4 and E4/E4 did not have sufficient number to be analyzed. E2/E2 was not detected. PPAR $\alpha$ L162V polymorphism results in three genotypes (Leu/Leu, Leu/Val, and Val/Val). Bold text indicates statistically significant differences.

analysis suggested that the presence of the lipoprotein lipase $\mathrm{H}+/ \mathrm{H}+$ genotype (adjusted $\mathrm{OR}=21.3 ; 95 \% \mathrm{CI}=2.32-335.3$; $P=0.003$ ), the PPAR $\alpha$ Leu/Val genotype (adjusted OR = 8.77; 95\% CI $=1.41-78.7 ; P=0.014)$, and elevated levels of VLDL-C (adjusted OR $=1.08 ; 95 \% \mathrm{CI}=1.01-1.17 ; P=0.023$ ) and TAG (adjusted $\mathrm{OR}=1.02 ; 95 \% \mathrm{CI}=1.00-1.03 ; P=0.021$ ) were associated with symptomatic $L$. infantum infection. In contrast, the HDL levels were inversely associated with symptomatic (adjusted OR $=0.84 ; 95 \% \mathrm{CI}=0.71-0.96 ; P=$ 0.006 ) and asymptomatic (adjusted $\mathrm{OR}=0.92 ; 95 \% \mathrm{CI}=$ 0.84-0.99; $P=0.027$ ) L. infantum infection (Table 4). When the lipoprotein levels were analyzed using the cut-off value established by selecting the point on the ROC curve that maximized the sensitivity and specificity for separating noninfected and infected symptomatic individuals, the association with symptomatic infection became more evident for elevated levels of VLDL-C (cut-off value $=33 \mathrm{mg} / \mathrm{dL}$; adjusted $\mathrm{OR}=18.8 ; 95 \% \mathrm{CI}=1.71-1014.4 ; P=0.009$ ) and TAG (cut-off value $=168 \mathrm{mg} / \mathrm{dL}$; adjusted $\mathrm{OR}=18.8 ; 95 \% \mathrm{CI}=1.71-1014.4$; $P=0.009)$.

\section{Discussion}

Discovering reliable markers for the assessment of individual and population risks of disease development is a major challenge for the scientific community. In this study, we evaluated potential markers of VL development. In areas that are endemic for VL, most infected individuals develop a protective immune response. Therefore, we focused on the nonimmune lipid elements as potential susceptibility factors based on the hypothesis that these alterations in VL patients may favor parasite growth and disease development. We further analyzed the polymorphisms of certain genes that may lead to such alterations in lipoprotein levels.

In a significant number of patients with clinical manifestations of VL, we found high TAG levels, high VLDL-C levels, and low HDL-C levels in infected symptomatic individuals 


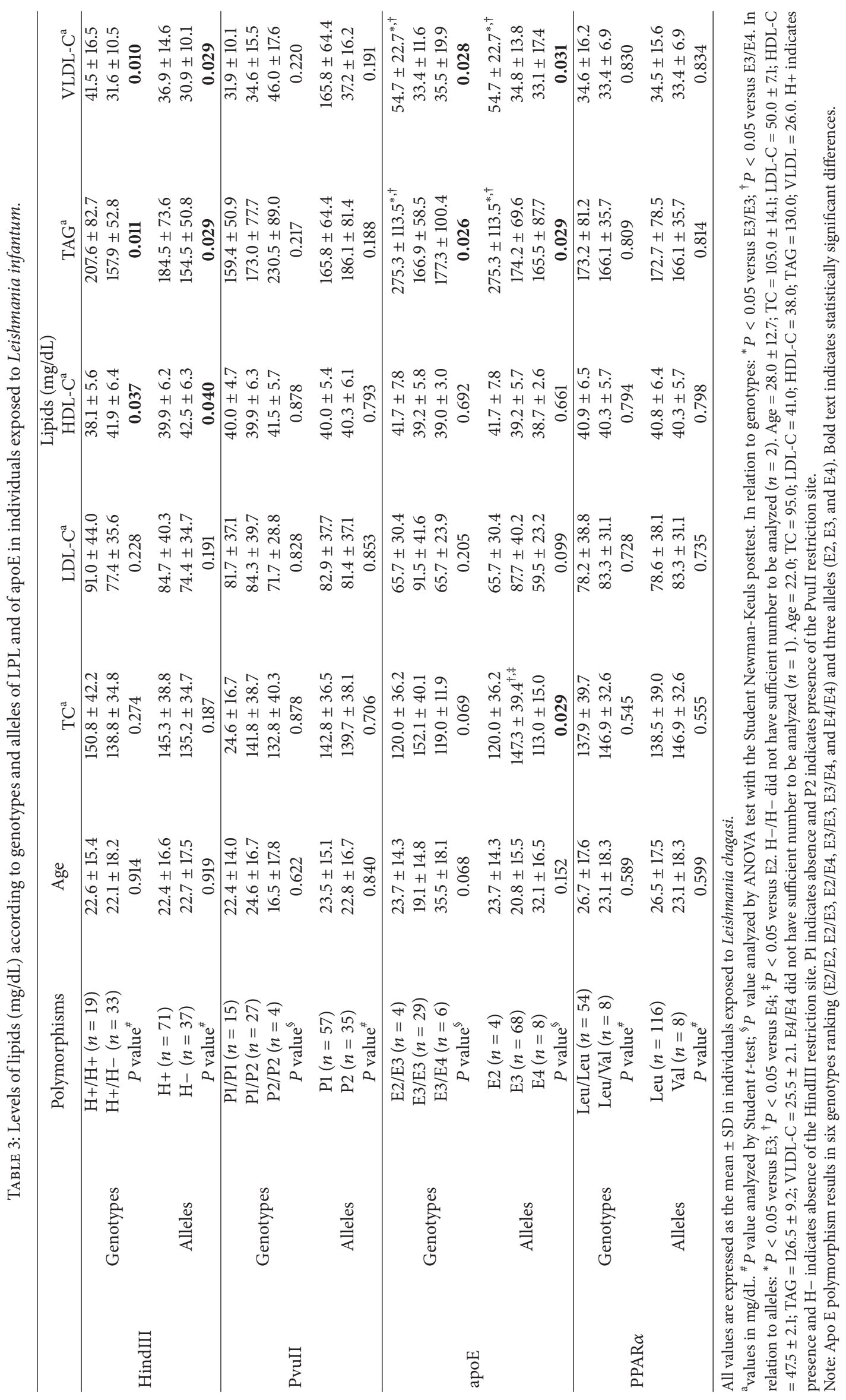


TABLE 4: Adjusted odds ratios (OR) and respective 95\% confidence intervals for the association between levels of lipids (mg/dL) and genotypes of LPL, apoE, and PPAR $\alpha$ and infected symptomatic and asymptomatic L. infantum infection.

\begin{tabular}{|c|c|c|c|c|c|c|}
\hline \multirow{2}{*}{ Variable } & \multicolumn{3}{|c|}{ Infected symptomatic infection } & \multicolumn{3}{|c|}{ Infected asymptomatic infection } \\
\hline & $\mathrm{OR}^{*}$ & $95 \% \mathrm{CI}^{*}$ & $P$ value & $\mathrm{OR}^{*}$ & $95 \% \mathrm{CI}^{*}$ & $P$ value \\
\hline \multicolumn{7}{|l|}{ HindIII } \\
\hline $\mathrm{H}-/ \mathrm{H}-$ & 1.00 & & & 1.00 & & \\
\hline $\mathrm{H}+/ \mathrm{H}-$ & 6.37 & $0.84-83.4$ & 0.083 & 2.04 & $0.49-10.4$ & 0.435 \\
\hline $\mathrm{H}+/ \mathrm{H}+$ & 21.3 & $2.32-335.3$ & 0.003 & 3.48 & $0.72-20.4$ & 0.143 \\
\hline \multicolumn{7}{|l|}{ PvuII } \\
\hline $\mathrm{P} 1 / \mathrm{P} 1$ & 1.00 & & & 1.00 & & \\
\hline $\mathrm{P} 1 / \mathrm{P} 2$ & 1.86 & $0.55-6.68$ & 0.395 & 1.42 & $0.52-3.98$ & 0.593 \\
\hline $\mathrm{P} 2 / \mathrm{P} 2$ & 0.71 & $0.10-5.28$ & 1.000 & 1.20 & $0.18-7.81$ & 1.000 \\
\hline \multicolumn{7}{|l|}{ ApoE } \\
\hline $\mathrm{E} 3 / \mathrm{E} 4$ & 1.00 & & & 1.00 & & \\
\hline E3/E3 & 2.66 & $0.52-16.1$ & 0.312 & 0.91 & $0.28-2.93$ & 1.000 \\
\hline E2/E3 & 4.31 & $0.23-113.7$ & 0.515 & 1.90 & $0.22-25.3$ & 0.825 \\
\hline \multicolumn{7}{|l|}{$\operatorname{PPAR} \alpha$} \\
\hline Leu/Leu & 1.00 & & & 1.00 & & \\
\hline Leu/Val & 8.77 & $1.41-78.7$ & 0.014 & 2.76 & $0.56-17.9$ & 0.272 \\
\hline TC & 0.99 & $0.97-1.01$ & 0.301 & 1.00 & 0.99-1.01 & 0.787 \\
\hline LDL-C & 0.99 & $0.97-1.01$ & 0.353 & 1.01 & $0.99-1.02$ & 0.414 \\
\hline HDL-C & 0.84 & $0.71-0.96$ & 0.006 & 0.92 & $0.84-0.99$ & 0.027 \\
\hline TAG & 1.02 & $1.00-1.03$ & 0.021 & 1.00 & $0.99-1.01$ & 0.597 \\
\hline VLDL-C & 1.08 & $1.01-1.17$ & 0.023 & 1.01 & $0.97-1.05$ & 0.580 \\
\hline
\end{tabular}

${ }^{*}$ Adjusted for age ( $\leq$ or $>20$ years) and gender.

$P$ values in bold indicate statistically significant differences.

as previously reported in the literature [19-22]. LPL, apoE, and $\operatorname{PPAR} \alpha$ are known as important components of lipid metabolism with roles in the homeostasis of different lipoprotein fractions and their transport, and the polymorphisms of these genes have been associated with cardiovascular diseases [28, 29, 34-36]. Therefore, these gene polymorphisms were analyzed in this study. We targeted LPL, apoE, and $\operatorname{PPAR} \alpha$ genes with polymorphisms and alleles that were analyzed in relation to symptomatic L. infantum infection. Importantly, an association between these polymorphisms and the expected phenotypes was observed, excluding the L162V polymorphism in the PPAR $\alpha$ gene. This finding is in contrast to most studies on gene polymorphisms in which the resulting phenotypes were unidentified. In the studied population, the $\mathrm{H}+/ \mathrm{H}+$ genotype and the $\mathrm{H}+$ allele were associated with elevated VLDL-C and TAG levels $(P<$ $0.05)$ and reduced HDL-C levels $(P<0.05)$. For the apoE genes and alleles, the genotype and expected phenotype association was unclear because the TAG and VLDL-C levels were significantly higher in individuals with the E2 allele than in individuals with the E3 and E4 alleles but not in individuals with the E3 allele. This finding may be due to the restricted number of samples and to the different roles of the six apoE allotypes. However, the association between the E2 allele and the alterations in lipoprotein levels may not have been uncovered because apoE2 carries significantly less VLDL cholesterol than apoE4 [35], and apoE2 impairs the lipolytic conversion of VLDL to LDL through inhibiting LPL activity [34].

The association between the $\mathrm{H}+/ \mathrm{H}+$ genotype and the $\mathrm{H}+$ allele with higher TAG and lower HDL-C levels was stronger in the infected symptomatic group than in the infected asymptomatic group. PvuII has been associated with variations in serum lipids in other diseases $[29,36]$; however, we did not detect any significant associations between the PvuII-restricted LPL genotypes and the presence of active VL or lipid fraction levels. This finding reinforces our suggestion that the HindIII-restricted LPL genotypes that were identified in this study are risk markers for the development of symptomatic L. infantum infection.

In the literature, high TAG and low HDL-C levels have been suggested as parameters for the diagnosis and followup of patients with VL [37]. However, in addition to a single study that suggested a role for LDL in the development of VL through tumor necrosis factor- $\alpha$ (TNF- $\alpha$ ) production [22], our study is the first to clearly find that the LPL genotype that leads to a phenotype of high VLDL-C levels, high TAG levels, and low HDL-C levels is a risk factor for active visceral leishmaniasis. Interestingly, we observed reduced highdensity lipoprotein cholesterol (HDL-C) levels in both the infected symptomatic and asymptomatic individuals compared with the noninfected individuals, which may suggest that decreased HDL levels initially favor infection. When TG and VLDL levels increase, progression to symptomatic infection may occur. 
The biological roles of VLDL and HDL in Leishmania infection need to be further analyzed; however, as an initial approach, we assessed the role of lipoprotein fractions in the mechanism of cell infection in vitro by examining the phagocytosis of promastigotes by macrophages. We observed $29 \%$ more parasites within the macrophages that were incubated for six hours with VLDL $(P<0.05)$ than in culture without the addition of lipoprotein. The coaddition of HDL significantly reduced (37\%) the parasite burden compared with $\operatorname{VLDL}(P<0.05)$.

The PPAR $\alpha$ L162V polymorphism has been demonstrated to be a risk marker for symptomatic infection; however, the mutated gene was not associated with altered lipoprotein levels. In a functional study of the Val-mutated PPAR $\alpha$ allele, this mutation in the DNA binding domain for PPAR $\alpha$ impacted the function of this transcription factor by preventing its proper binding to the region of DNA that is under its transcriptional control [38]. The frequency of this mutated allele was significantly higher in individuals who presented with symptomatic infection; therefore, the inefficient activation of lipid metabolism genes may lead to higher serum levels of lipid fractions. However, the results of this study did not indicate a relationship between the polymorphisms and the lipid profiles. A possible explanation for this finding is the low number of individuals who presented with the Val allele. This variant allele is relatively rare (an observed frequency of $10.8 \%$ ), and the lipid profiles of only 8 individuals who were carrying this allele were measured. Therefore, any statistical evidence for a relationship between this mutation and the lipid profiles would be unlikely. Besides the PPAR $\alpha$ gene can modulate lipid pathways, which may not be reflected in serum-lipid levels, and indirectly affect the outcome of Leishmania infantum infection. In addition, PPAR $\alpha$ is known for its major role in the upregulation of cholesterol trafficking and efflux in macrophages [39]. Interestingly, in transcriptomic studies with Leishmania-infected mouse macrophages, cholesterol accumulation was clearly observed within host cells, which was achieved by the upregulation of genes that are implicated in the uptake of LDL (CD36 and the LDL receptor) and by the downregulation of genes that mediate cholesterol efflux (ABCA1 and CYP27) [40, 41]. In this scenario, a PPAR $\alpha$ variant allele may contribute to the modulation of infection by enhancing the accumulation of cholesterol in infected cells and by shuttling cholesterol to Leishmania parasites. We cannot rule out either the modulation of infection by PPAR $\alpha$ functions in inflammatory processes [39].

The results of this study and those from two previous studies $[30,42]$ support the presupposition that the genotypes for molecules that are not related to the adaptive immune system, that is, LPL, PPAR $\alpha$, and mannan-binding lectin (MBL) genotypes, have a significant correlation with $L$. infantum infection or disease. Notably, in addition to the roles of MBL in the complement system [43] and the autoimmune process [44], MBL levels are affected by the activation of $\operatorname{PPAR} \alpha$ [45], which induces LPL expression [46].

These genetic markers and the more easily accessible lipid levels may be used to evaluate the risk of $L$. infantum infection and disease in endemic areas and may be used to follow up patients who present with active-phase infection. Furthermore, our results suggest that we should consider studying lipid-lowering drugs as a complementary treatment for VL patients.

\section{Conflict of Interests}

The authors declare that there is no conflict of interests regarding the publication of this paper.

\section{Authors' Contribution}

Márcia Dias Teixeira Carvalho and Diego Peres Alonso and the senior authors Paulo Eduardo Martins Ribolla and Hiro Goto had, respective equal participation in this study.

\section{Acknowledgments}

The authors would like to acknowledge the Conselho Nacional de Desenvolvimento Científico e Tecnológico (Grant 154581/2006-2, research fellowship to H. Goto), the Fundação de Amparo à Pesquisa do Estado de São Paulo (Fellowship 06/60006-9 to M, Dias Teixeira Carvalho), and the Laboratório de Investigação Médica (LIM/38 HC-FMUSP). They also would like to thank Valéria Sutti Nunes, Maria das Graças Prianti, Letícia Amaral Nogueira Alonso, and Débora Colombi for providing laboratory assistance and all of the study participants for agreeing to contribute to this research. In addition, they would like to thank Isac Castro for the excellent statistical assistance and are grateful to the Laboratório de Lípides (LIM/10 HC-FMUSP) for its assistance.

\section{References}

[1] I. L. Mauricio, M. W. Gaunt, J. R. Stothard, and M. A. Miles, "Genetic typing and phylogeny of the Leishmania donovani complex by restriction analysis of PCR amplified gp63 intergenic regions," Parasitology, vol. 122, no. 4, pp. 393-403, 2001.

[2] R. Badaro, T. C. Jones, R. Lorenco et al., "A prospective study of visceral leishmaniasis in an endemic area of Brazil," Journal of Infectious Diseases, vol. 154, no. 4, pp. 639-649, 1986.

[3] H. Goto and M. D. G. Prianti, "Immunoactivation and immunopathogeny during active visceral leishmaniasis," Revista do Instituto de Medicina Tropical de Sao Paulo, vol. 51, no. 5, pp. 241-246, 2009.

[4] B. Bucheton, L. Abel, M. M. Kheir et al., "Genetic control of visceral leishmaniasis in a Sudanese population: candidate gene testing indicates a linkage to the NRAMP1 region," Genes \& Immunity, vol. 4, no. 2, pp. 104-109, 2003.

[5] H. S. Mohamed, M. E. Ibrahim, E. N. Miller et al., "SLC11A1 (formerly NRAMP1) and susceptibility to visceral leishmaniasis in the Sudan," European Journal of Human Genetics, vol. 12, no. 1, pp. 66-74, 2004.

[6] H. S. Mohamed, M. E. Ibrahim, E. N. Miller et al., "Genetic susceptibility to visceral leishmaniasis in The Sudan: linkage and association with IL4 and IFNGR1," Genes and Immunity, vol. 4, no. 5, pp. 351-355, 2003. 
[7] T. M. Karplus, S. M. B. Jeronimo, H. Chang et al., "Association between the tumor necrosis factor locus and the clinical outcome of Leishmania chagasi infection," Infection and Immunity, vol. 70, no. 12, pp. 6919-6925, 2002.

[8] B. Bucheton, L. Abel, S. El-Safi et al., "A major susceptibility locus on chromosome 22q12 plays a critical role in the control of kala-azar," American Journal of Human Genetics, vol. 73, no. 5, pp. 1052-1060, 2003.

[9] B. Bucheton, L. Argiro, C. Chevillard et al., "Identification of a novel G245R polymorphism in the IL-2 receptor $\beta$ membrane proximal domain associated with human visceral leishmaniasis," Genes and Immunity, vol. 8, no. 1, pp. 79-83, 2007.

[10] A. Moravej, M. Rasouli, S. Asaei, M. Kalani, and Y. Mansoori, "Association of interleukin-18 gene variants with susceptibility to visceral leishmaniasis in Iranian population," Molecular Biology Reports, vol. 40, no. 6, pp. 4009-4014, 2013.

[11] A. Moravej, M. Rasouli, M. Kalani et al., "IL-1 $\beta$ (-511T/C) gene polymorphism not IL- $\beta(+3953 \mathrm{~T} / \mathrm{C})$ and LT $-\alpha(+252 \mathrm{~A} / \mathrm{G})$ gene variants confers susceptibility to visceral leishmaniasis," Molecular Biology Reports, vol. 39, no. 6, pp. 6907-6914, 2012.

[12] J. M. Blackwell, M. Fakiola, M. E. Ibrahim et al., "Genetics and visceral leishmaniasis: Of mice and man," Parasite Immunology, vol. 31, no. 5, pp. 254-266, 2009.

[13] M. Hajilooi, K. Sardarian, M. Dadmanesh et al., "Is the IL10 -819 polymorphism associated with visceral leishmaniasis?" Inflammation, pp. 1-6, 2013.

[14] S. Mehrotra, M. Fakiola, J. Oommen et al., "Genetic and functional evaluation of the role of CXCR1 and CXCR2 in susceptibility to visceral leishmaniasis in North-East India," BMC Medical Genetics, vol. 12, article 162, 2011.

[15] S. Mehrotra, M. Fakiola, A. Mishra et al., "Genetic and functional evaluation of the role of DLL1 in susceptibility to visceral leishmaniasis in India," Infection, Genetics and Evolution, vol. 12, no. 6, pp. 1195-1201, 2012.

[16] S. Mehrotra, J. Oommen, A. Mishra et al., "No evidence for association between SLC11A1 and visceral leishmaniasis in India," BMC Medical Genetics, vol. 12, article 71, 2011.

[17] M. Fakiola, A. Strange, H. J. Cordell et al., "Common variants in the HLA-DRB1-HLA-DQA1 HLA class II region are associated with susceptibility to visceral leishmaniasis," Nature Genetics, vol. 45, no. 2, pp. 208-213, 2013.

[18] C. R. Davies and A. S. M. Gavgani, "Age, acquired immunity and the risk of visceral leishmaniasis: a prospective study in Iran," Parasitology, vol. 119, no. 3, pp. 247-257, 1999.

[19] E. D. Bekaert, E. Dole, D. Y. Dubois et al., "Alterations in lipoprotein density classes in infantile visceral Leishmaniasis: presence of apolipoprotein SAA," European Journal of Clinical Investigation, vol. 22, no. 3, pp. 190-199, 1992.

[20] E. D. Bekaert, R. Kallel, M.-. Bouma et al., "Plasma lipoproteins in infantile visceral Leishmaniasis: deficiency of apolipoproteins A-I and A-II," Clinica Chimica Acta, vol. 184, no. 2, pp. 181-191, 1989.

[21] C. S. Lal, A. Kumar, S. Kumar et al., "Hypocholesterolemia and increased triglyceride in pediatric visceral leishmaniasis," Clinica Chimica Acta, vol. 382, no. 1-2, pp. 151-153, 2007.

[22] N. M. Soares, T. F. Leal, M. C. Fiúza et al., "Plasma lipoproteins in visceral leishmaniasis and their effect on Leishmaniainfected macrophages," Parasite Immunology, vol. 32, no. 4, pp. 259-266, 2010.

[23] D. Rosenzweig, D. Smith, F. Opperdoes, S. Stern, R. W. Olafson, and D. Zilberstein, "Retooling Leishmania metabolism: From sand fly gut to human macrophage," The FASEB Journal, vol. 22, no. 2, pp. 590-602, 2008.

[24] H. Jansen, B. Breedveld, and K. Schoonderwoerd, "Role of lipoprotein lipases in postprandial lipid metabolism," Atherosclerosis, vol. 141, no. 1, pp. S31-S34, 1998.

[25] M. D. T. Carvalho, L. M. Harada, M. Gidlund, D. F. J. Ketelhuth, P. Boschcov, and E. C. R. Quintão, "Macrophages take up triacylglycerol-rich emulsions at a faster rate upon coincubation with native and modified LDL: an investigation on the role of natural chylomicrons in atherosclerosis," Journal of Cellular Biochemistry, vol. 84, no. 2, pp. 309-323, 2001.

[26] M. Emi, L. L. Wu, M. A. Robertson et al., "Genotyping and sequence analysis of apolipoprotein E isoforms," Genomics, vol. 3, no. 4, pp. 373-379, 1988.

[27] C. Duval, M. Muller, and S. Kersten, "PPARalpha and dyslipidemia," Biochimica et Biophysica Acta, vol. 1771, no. 8, pp. 961971, 2007.

[28] Y. I. Ahn, M. I. Kamboh, R. F. Hamman, S. A. Cole, and R. E. Ferrell, "Two DNA polymorphisms in the lipoprotein lipase gene and their associations with factors related to cardiovascular disease," Journal of Lipid Research, vol. 34, no. 3, pp. 421-428, 1993.

[29] E. Socquard, A. Durlach, C. Clavel, P. Nazeyrollas, and V. Durlach, "Association of HindIII and PvuII genetic polymorphisms of lipoprotein lipase with lipid metabolism and macrovascular events in type 2 diabetic patients," Diabetes \& Metabolism, vol. 32, no. 3, pp. 262-269, 2006.

[30] D. P. Alonso, A. F. B. Ferreira, P. E. M. Ribolla et al., "Genotypes of the mannan-binding lectin gene and susceptibility to visceral leishmaniasis and clinical complications," Journal of Infectious Diseases, vol. 195, no. 8, pp. 1212-1217, 2007.

[31] T. G. Kirchgessner, J.-. Chuat, C. Heinzmann et al., "Organization of the human lipoprotein lipase gene and evolution of the lipase gene family," Proceedings of the National Academy of Sciences of the United States of America, vol. 86, no. 24, pp. 96479651, 1989.

[32] K. Oka, G. T. Tkalcevic, J. Stocks, D. J. Galton, and W. V. Brown, "Nucleotide sequence of PvuII polymorphic site at the human lipoprotein lipase gene locus," Nucleic Acids Research, vol. 17, no. 16, p. 6752, 1989.

[33] R. D. Santos, "III Brazilian Guidelines on Dyslipidemias and Guideline of Atherosclerosis Prevention from Atherosclerosis Department of Sociedade Brasileira de Cardiologia," Arquivos Brasileiros de Cardiologia, vol. 77, supplement 3, pp. 1-48, 2001.

[34] Y. Huang, X. Q. Liu, S. C. Rail Jr., and R. W. Mahley, "Apolipoprotein E2 reduces the low density lipoprotein level in transgenic mice by impairing lipoprotein lipase-mediated lipolysis of triglyceride-rich lipoproteins," The Journal of Biological Chemistry, vol. 273, no. 28, pp. 17483-17490, 1998.

[35] L. Dong and K. H. Weisgraber, "Human apolipoprotein E4 domain interaction. Arginine 61 and glutamic acid 255 interact to direct the preference for very low density lipoproteins," Journal of Biological Chemistry, vol. 271, no. 32, pp. 19053-19057, 1996.

[36] J. L. Anderson, G. J. King, T. L. Bair et al., "Association of lipoprotein lipase gene polymorphisms with coronary artery disease," Journal of the American College of Cardiology, vol. 33, no. 4, pp. 1013-1020, 1999.

[37] G. Seçmeer, A. B. Cengiz, A. Gürgey et al., "Hypertriglyceridemia and decreased high-density lipoprotein could be a clue for visceral leishmaniasis," Infectious Diseases in Clinical Practice, vol. 14, no. 6, pp. 401-402, 2006. 
[38] A. Sapone, J. M. Peters, S. Sakai et al., "The human peroxisome proliferator-activated receptor $\alpha$ gene: Identification and functional characterization of two natural allelic variants," Pharmacogenetics, vol. 10, no. 4, pp. 321-333, 2000.

[39] E. Rigamonti, G. Chinetti-Gbaguidi, and B. Staels, "Regulation of macrophage functions by PPAR- $\alpha$, PPAR- $\gamma$, and LXRs in mice and men," Arteriosclerosis, Thrombosis, and Vascular Biology, vol. 28, no. 6, pp. 1050-1059, 2008.

[40] J. Osorio y Fortéa, E. de La Llave, B. Regnault et al., “Transcriptional signatures of $\mathrm{BALB} / \mathrm{c}$ mouse macrophages housing multiplying Leishmania amazonensis amastigotes," BMC Genomics, vol. 10, article 119, 2009.

[41] I. Rabhi, S. Rabhi, R. Ben-Othman et al., "Transcriptomic signature of Leishmania infected mice macrophages: a metabolic point of view," PLoS Neglected Tropical Diseases, vol. 6, no. 8, Article ID e1763, 2012.

[42] S. Hamdi, R. Ejghal, M. Idrissi et al., "A variant in the promoter of MBL2 is associated with protection against visceral leishmaniasis in Morocco," Infection, Genetics and Evolution, vol. 13, no. 1, pp. 162-167, 2013.

[43] W. K. Eddie Ip, K. Takahashi, R. Alan Ezekowitz, and L. M. Stuart, "Mannose-binding lectin and innate immunity," Immunological Reviews, vol. 230, no. 1, pp. 9-21, 2009.

[44] S. Saevarsdottir, T. Vikingsdottir, and H. Valdimarsson, "The potential role of mannan-binding lectin in the clearance of self-components including immune complexes," Scandinavian Journal of Immunology, vol. 60, no. 1-2, pp. 23-29, 2004.

[45] M. Rakhshandehroo, R. Stienstra, N. J. de Wit et al., "Plasma mannose-binding lectin is stimulated by PPAR $\alpha$ in humans," The American Journal of Physiology-Endocrinology and Metabolism, vol. 302, no. 5, pp. E595-E602, 2012.

[46] M. Panadero, C. Bocos, and E. Herrera, "Relationship between lipoprotein lipase and peroxisome proliferator-activated receptor- $\alpha$ expression in rat liver during development," Journal of Physiology and Biochemistry, vol. 62, no. 3, pp. 189-198, 2006. 


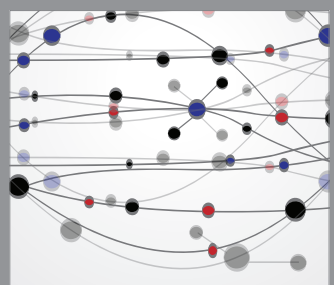

The Scientific World Journal
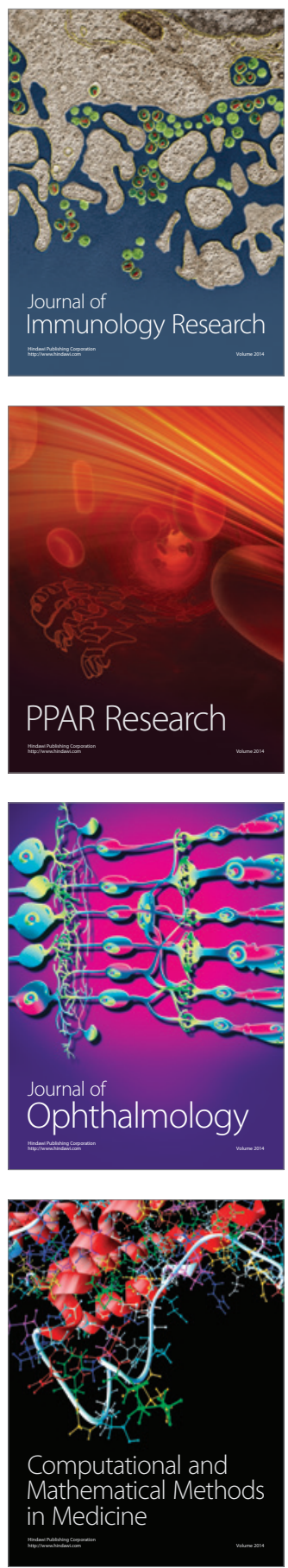

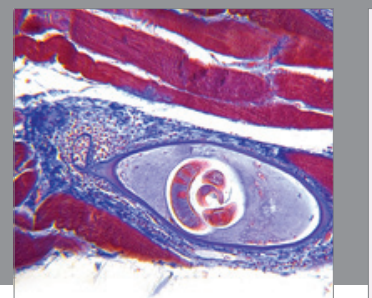

Gastroenterology

Research and Practice
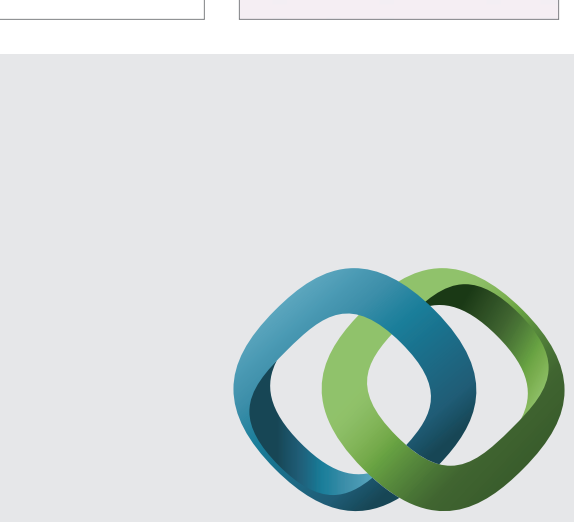

\section{Hindawi}

Submit your manuscripts at

http://www.hindawi.com
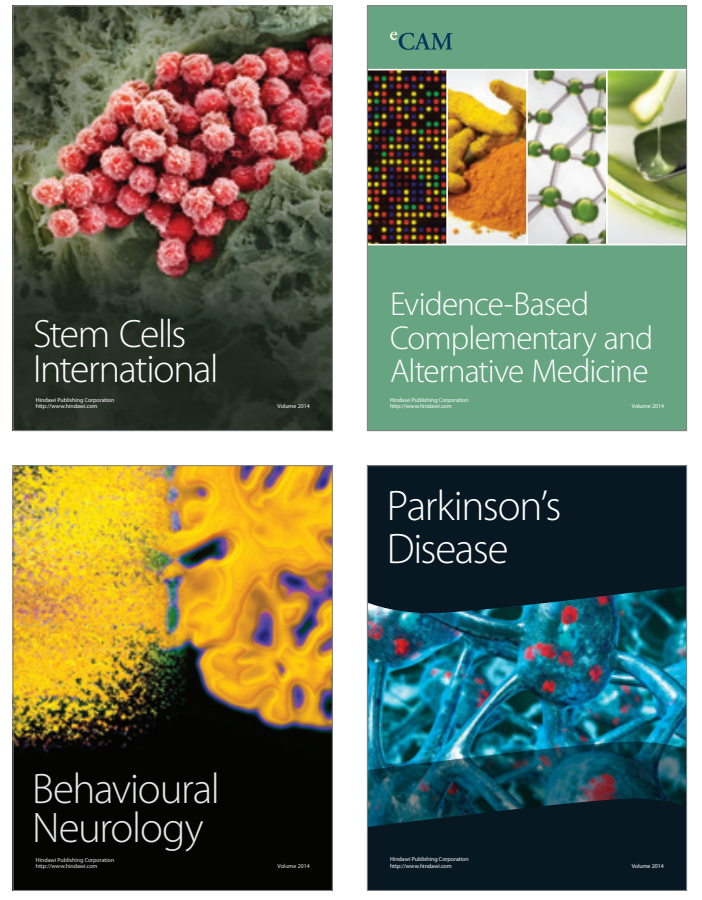
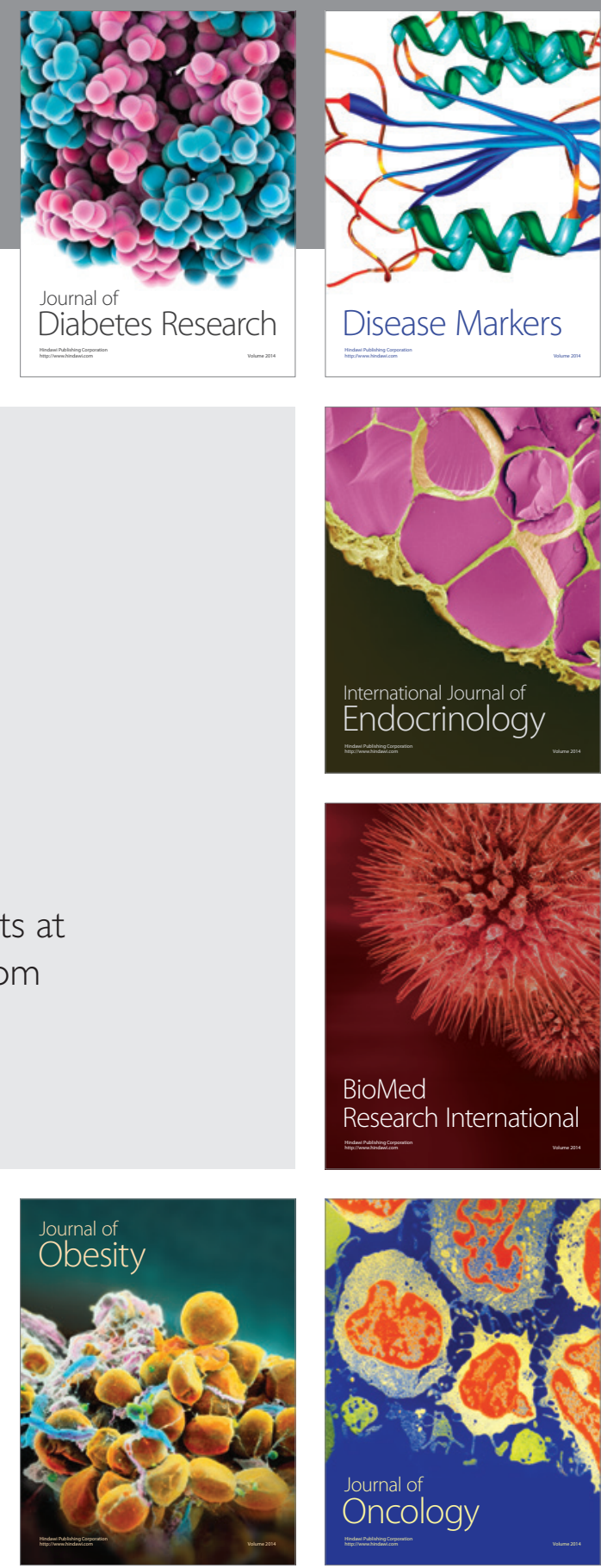

Disease Markers
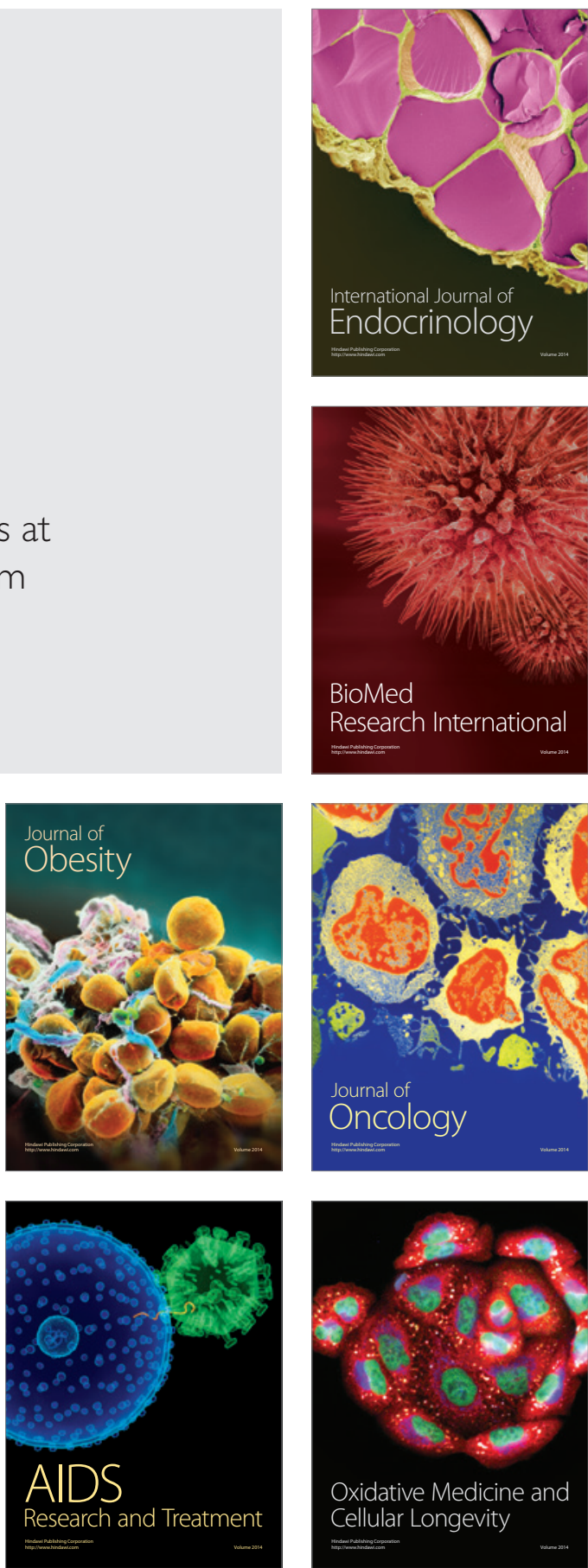